\title{
Phase II multicenter trial of docetaxel, epirubicin, and 5-fluorouracil (DEF) in the treatment of advanced gastric cancer: a novel, safe, and active regimen
}

\author{
André M. Murad ${ }^{1}$, Nils G. Skare 2 , Jéferson Vinholes 3 , Sérgio Lago ${ }^{4}$, and Ricardo Pecego ${ }^{5}$; Brazilian Gastric \\ Cancer Study Group, Belo Horizonte, MG, Brazil \\ ${ }^{1}$ Oncology Department, Hospital das Clinicas Universidade Federal de Minas Gerais, Rua Piaui 150, Belo Horizonte, MG Brazil, $30150-320$ \\ ${ }^{2}$ Hospital Erasto Gaertner, Centro de Projetos de Ensino e Pesquisa, Liga Paranaense de Combate ao Câncer, Curitiba, PR, Brazil \\ ${ }^{3}$ Irmandade Santa Casa de Misericórdia de Porto Alegre, Unidade de Apoio à Pesquisa, Porto Alegre, RS, Brazil \\ ${ }^{4}$ Hospital São Lucas - PUC-RGS, Porto Alegre, RS, Brazil \\ ${ }^{5}$ Hospital das Clínicas da UFG, Goiânia, GO, Brazil
}

\begin{abstract}
Background. This study evaluated the efficacy and safety of docetaxel, epirubicin, and 5-fluorouracil (5-FU) [DEF] as treatment for locally advanced unresectable or metastatic gastric cancer.

Methods. Thirty-seven patients participated in the study (median age, 56 years; range, 22-73 years); Eastern Cooperative Oncology Group performance status [PS], 0-2). Docetaxel $75 \mathrm{mg} / \mathrm{m}^{2}$ IV (day 1), 5-FU $500 \mathrm{mg} / \mathrm{m}^{2}$ IV (days 1-3), and epirubicin $50 \mathrm{mg} / \mathrm{m}^{2}$ IV (day 1) were administered every 3 weeks for six cycles.

Results. In total, $20 / 37$ patients $(54 \%)$ completed six treatment cycles. Thirteen patients $(35 \%$; $95 \%$ confidence intervals $[\mathrm{CI}], \mathbf{2 0} \%$ to $51 \%$ ) had an objective response; 1 patient (3\%) achieved a complete response and 12 patients $(32 \%)$ achieved partial responses. Stable disease was observed in 7 patients $(19 \%)$ and progressive disease in 5 patients $(14 \%)$. Twelve patients $(32 \%)$ were unevaluable. Clinical benefit (based on PS, weight gain, and analgesic consumption) was observed in 11 patients $(30 \%)$. Median follow-up was 41 months (range, 26-53 months), median time to progression was 6.6 months (range, 0.5-29.2 months), median overall survival was 10.7 months (range, 7.0-14.6 months), and 1-year survival was $40 \%$. The regimen was well tolerated. Grade 3-4 febrile neutropenia occurred in 8 patients $(22 \% ; 6 \%$ of cycles) and grade 3-4 neutropenia in 1 patient (1\% of cycles). The most frequent grade 3-4 toxicities were alopecia (11\% of cycles), diarrhea ( $4 \%$ of cycles) and vomiting ( $2 \%$ of cycles); grade 1-2 asthenia and fatigue occurred in $43 \%$ of cycles. Conclusion. DEF is effective in the treatment of advanced gastric cancer, and has a good safety profile.
\end{abstract}

Offprint requests to: A.M. Murad

Presented at: 2002 ASCO Meeting (Poster Section): Proc Am Soc Clin Oncol 2002;21:163a and 2002 ESMO Meeting (Poster Section): Ann Oncol 2002;13(Suppl 5):192.

Received: August 22, 2005 / Accepted: January 10, 2006
Key word Docetaxel · Epirubicin · 5-Fluorouracil · Gastric cancer

\section{Introduction}

Gastric adenocarcinoma is the second leading cause of cancer worldwide. It occurs twice as often in men as in women, and is more frequent in the elderly, with a mean age at diagnosis of 70-73 years. As most cases are diagnosed at an advanced stage, the prognosis for this disease is extremely poor, with a 5-year survival of $5 \%-15 \%$ [1]. In Brazil, the incidence and mortality rates for gastric cancer are particularly alarming. According to the Brazilian Ministry of Health, 22150 new cases of gastric cancer were expected in 2003 [2]. As the second leading cause of cancer-related death in Brazil, there were, in fact, 11550 reported deaths in 2003 [2].

Surgery is the only potentially curative treatment for localized gastric cancer. Chemotherapy, which may or may not be administered with radiotherapy, is used to treat advanced or metastatic disease, and the efficacy of chemotherapy with palliative intent is now widely accepted [3].

5-Fluorouracil (5-FU) is effective and widely used in the treatment of advanced gastric cancer, producing a response rate of approximately $20 \%$, with manageable toxicity [3]. Overall survival of 5-7 months has been reported for 5-FU monotherapy in phase III randomized studies [4,5], and all current reference combination regimens in advanced gastric cancer contain 5-FU. Many different combinations have been widely used in advanced gastric cancer, such as 5-FU, doxorubicin, and mitomycin (FAM); 5-FU, doxorubicin, and high-dose methotrexate (FAMTX); etoposide, doxorubicin, and 
cisplatin (EAP); etoposide, leucovorin, and 5-FU (ELF); epirubicin, cisplatin, and 5-FU continuous infusion (ECF); cisplatin, epirubicin, leucovorin, and 5-FU (PELF); and several regimens of cisplatin and 5-FU [6]. In randomized studies, FAMTX, cisplatin/5-FU, and PELF have been demonstrated to be more effective than FAM [7-9]; median survival was also significantly greater with FAMTX versus FAM [8]. FAMTX showed similar responses rates to EAP [10] and cisplatin/5-FU and ELF [11], with no significant differences in median survival. ECF was reported to be more active than FAMTX [12].

Despite response rates of up to $51 \%$ in these trials, the median survival in patients with advanced disease was consistently less than 10 months. Moreover, toxicities such as leukopenia or alopecia are often present in cisplatin- or etoposide-based regimens.

There is a clear necessity for new drugs and new therapeutic interventions to be studied in order that response rates and survival can be improved. The taxanes, docetaxel and paclitaxel, are one of the most promising groups of cytotoxic agents in clinical use expressing good antitumor activity, particularly in adenocarcinomas such as breast, lung, and ovarian cancers [13]. Positive results have been achieved with both paclitaxel [14-16] and docetaxel [17-22] as single agents in the treatment of gastric cancer: in phase II trials, overall response rates of $17 \%-32 \%$ have been reported with a 3-weekly regimen of paclitaxel (doses ranging from 200 to $250 \mathrm{mg} / \mathrm{m}^{2}$ ) [14-16] and $17 \%-24 \%$ with 3weekly docetaxel (doses ranging from 60 to $100 \mathrm{mg} / \mathrm{m}^{2}$ ) [17-22]. Higher overall response rates have been achieved using taxane-based combination regimens, usually with platinum and/or 5-FU [23-28]; overall response rates of $32 \%-66 \%$ have been observed with paclitaxel combination therapies $[23,29,30]$ and rates of $33 \%-60 \%$ with docetaxel combination regimens [2428].

In the present trial, we studied the efficacy and safety of docetaxel $75 \mathrm{mg} / \mathrm{m}^{2}$ intravenous (IV), epirubicin $50 \mathrm{mg} / \mathrm{m}^{2}$ IV and 5 -FU $500 \mathrm{mg} / \mathrm{m}^{2}$ IV (DEF) in the treatment of advanced gastric cancer. Epirubicin was chosen over doxorubicin as, in addition to being a relatively less toxic anthracycline, in vitro data from human tumor cells suggest that epirubicin is more active than doxorubicin against gastric cancer cells [31]. The underlying rationale for the proposed triplet regimen is that it combines three drugs with different mechanisms of action, providing efficacy with almost no overlapping toxicity.

\section{Patients and methods}

\section{Patient selection}

To be eligible for this study, patients had to have: pathologically confirmed, nonresectable locally advanced or metastatic malignant gastric cancer; at least one measurable lesion in a nonirradiated area; not received prior chemotherapy; an Eastern Cooperative Oncology Group (ECOG) performance status (PS) of 2 or less; age 18-75 years; life expectancy of 12 weeks or more; and adequate hepatic, renal, and bone marrow function.

Patients were excluded from the study if they experienced symptomatic peripheral neuropathy of National Cancer Institute Common Toxicity Criteria (NCI-CTC, version 2.0) [32] grade 2 or more; were pregnant or breastfeeding or were of child-bearing potential without using adequate contraception; had any other current or prior malignancy (with the exception of excised cervical carcinoma in situ or squamous cell skin carcinoma treated by surgery only); showed clinical evidence of major organ failure; had central nervous system (CNS) metastases; had bone metastases as the sole disease site; had active uncontrolled infection or disease; had a neurologic or mental disease not consistent with adequate comprehension of the patient information sheet; or were receiving concurrent treatment with any other drugs that could potentially interfere with the study evaluation (such as longterm administration of corticosteroids). The local ethics committee approved the protocol, and written informed consent was obtained from all patients.

\section{Study design}

In this multicenter, open-label, phase II trial, patients received DEF, i.e., docetaxel $75 \mathrm{mg} / \mathrm{m}^{2}$ (60-min IV infusion) on day 1 , epirubicin $50 \mathrm{mg} / \mathrm{m}^{2}$ (15-min IV infusion) on day 1 , and 5 -FU $500 \mathrm{mg} / \mathrm{m}^{2}$ (15-min IV infusion) on days 1,2 , and 3 . Patients were treated on an outpatient basis and premedicated with dexamethasone $8 \mathrm{mg}$ orally to be given the night before and on the morning of the docetaxel infusion and $1 \mathrm{~h}$ before each docetaxel infusion. Additionally, dexamethasone was given on the night of chemotherapy administration and in the morning and evening of the day after the end of the docetaxel infusion. Ondansetron $8 \mathrm{mg}$ was given before epirubicin administration and metoclopramide $20 \mathrm{mg}$ was given before 5-FU administration.

Cycles were repeated every 3 weeks for six cycles, unless progressive disease or unacceptable toxicity occurred, or patient consent was withdrawn. In the event of toxicity (NCI-CTC definitions), the following dose reductions and treatment delays were planned. In the 
case of insufficient hematologic function (neutrophil count $<1500 / \mathrm{mm}^{3}$; platelet count $<100000 / \mathrm{mm}^{3}$ ) on day 21 of any cycle, treatment was delayed for up to 14 days. If recovery did not occur at this point, treatment was discontinued. Patients with febrile neutropenia or a delay in therapy as a result of myelosuppression were to be treated with granulocyte colony-stimulating factor (G-CSF) on subsequent cycles.

\section{Dose modifications}

In the case of grade 4 thrombocytopenia or subsequent febrile neutropenia not controlled with G-CSF, docetaxel was reduced by $20 \%$. An additional docetaxel dose reduction $(20 \%)$ was conducted upon a third episode of febrile neutropenia. If further febrile neutropenia occurred, treatment was discontinued. Patients who developed hepatic function abnormalities during therapy had the dose of docetaxel reduced by $20 \%$ if there was either an increase in aspartate aminotransferase (AST) or alanine aminotransferase (ALT) (2.5-5 times upper limit of normal [ULN]) with alkaline phosphatase $(\leq 2.5$ times ULN) or if alkaline phosphatase was 2.5-5 times ULN and AST or ALT were 1.5-5 times ULN. Elevations of any of these enzymes ( $>5$ times ULN) resulted in docetaxel being withheld for a maximum of 2 weeks; the patient was withdrawn from the study if recovery did not occur in that timeframe. Grade 3 skin toxicity, diarrhea, or mucositis also indicated docetaxel, epirubicin, and 5-FU dose reductions of $20 \%$. Grade 4 hematologic toxicity resulted in the patient being withdrawn from the study.

\section{Study evaluations}

In the week preceding treatment, patients underwent a complete medical history, physical examination, and electrocardiogram (ECG). A chest radiograph, computed tomography (CT) scan, magnetic resonance imaging (MRI; if indicated), evaluation of nonmeasurable lesions, and endoscopy (for locally advanced disease) were carried out in the 4 weeks preceding the initiation of treatment. Biologic parameters (blood cell count, serum creatinine, bilirubin, AST, ALT, and alkaline phosphatase) were measured at baseline and before each treatment cycle. Blood cell count was also measured on day 8 of each cycle.

All adverse events were graded using the NCI-CTC criteria (version 2) at each cycle. Tumor evaluation was carried out at the end of the second and the sixth cycles, according to standard World Health Organization (WHO) criteria [33], with appropriate clinical and radiologic examinations, and responses were to be confirmed within 4 weeks. The clinical benefit of the regimen was assessed using a modified version of the system developed to assess clinical benefit responses in patients receiving treatment for advanced pancreatic adenocarcinoma [34]. Evaluation of responses involved three components: pain (based on analgesic consumption and pain intensity measured by analog nonvisual scale), ECOG PS, and weight gain.

\section{Clinical response}

Each parameter was measured at baseline and then regularly (every two cycles) during the study. Clinical benefit response was defined as a sustained improvement ( $>4$ weeks) in at least one parameter without a worsening of any other. The intent-to-treat (ITT) population consisted of all patients enrolled in the study. All efficacy and safety analyses were conducted using the ITT population. The overall response rate was calculated with $95 \%$ confidence intervals (CI). Progression was defined as clinical progression or death due to any cause, and the progression and survival times were measured from the time of study entry until the occurrence of either event. Overall survival (OS) was measured from the initial treatment until death. These values were estimated using the Kaplan-Meier method.

\section{Results}

\section{Patient characteristics}

Between November 1999 and January 2002, 37 patients were enrolled in the study at five Brazilian centers.

Patient characteristics are listed in Table 1 . Twentyfive $(68 \%)$ patients were male and $12(32 \%)$ were female. Most patients had a good PS $(83 \%$ had an ECOG PS of 0 or 1). All patients had histologically confirmed adenocarcinomas, the majority of which were poorly or moderately differentiated ( $32 \%$ and $35 \%$, respectively), and distant metastases were identified in $68 \%$ of patients. One patient had a resectable tumor and was considered a protocol deviation, as was another patient with a PS of 3 .

\section{Efficacy}

Of the 37 patients, 12 (32\%) were not assessed for response due to: informed consent withdrawal (4 patients), discontinuation due to adverse events (4 patients), early death ( 2 patients; 1 due to pneumonia and 1 due to septicemia), and protocol deviation (2 patients). These 12 patients were included in the ITT analysis of response rate, progression-free survival, and overall survival. Response rates are shown in Table 2. There were 13 (35\%; 95\% CI, $20 \%$ to $51 \%$ ) objective responses, including $1(3 \%)$ complete response and 12 $(32 \%)$ partial responses. 
Table 1. Patient characteristics, ITT population $(n=37)$

\begin{tabular}{lc} 
Characteristic & $n(\%)$ \\
\hline Sex & \\
$\quad$ Male & $25(68)$ \\
$\quad$ Female & $12(32)$ \\
Median age, years (range) & $56(22-73)$ \\
ECOG performance status & \\
0 & $9(24)$ \\
1 & $22(59)$ \\
2 & $5(14)$ \\
3 a & $1(3)$ \\
Histopathologic differentiation ${ }^{\text {b }}$ & \\
Adenocarcinoma & 0 \\
$\quad$ Grade I & $13(35)$ \\
$\quad$ Grade II & $12(32)$ \\
$\quad$ Grade III & $3(8)$ \\
Undifferentiated & $9(24)$ \\
Not specified & $12(32)$ \\
Staging & $25(68)$ \\
$\quad$ Locally advanced & \\
Metastatic & \\
\hline ECOG, Eastern Cooperative Oncology Group; ITT, intent to treat; \\
TNM, tumor node metastases \\
a Protocol deviation \\
${ }^{b}$ Laurén (1965)
\end{tabular}

Table 2. Response rates, ITT population $(n=37)$

\begin{tabular}{lrc}
\hline Response & $n(\%)$ & $95 \%$ CI \\
\hline Complete response & $1(3)$ & - \\
Partial response & $12(32)$ & - \\
Stable disease & $7(19)$ & - \\
Progressive disease & $5(14)$ & - \\
Not assessable & $12(32)$ & 20 to 51 \\
Overall response rate & $13(35)$ &
\end{tabular}

CI, confidence intervals; ITT, intent to treat
Clinical benefit was observed in $11(30 \%)$ patients. In 2 patients, all three parameters (pain, ECOG PS reduction, and weight gain) improved. In 5 patients, two parameters were improved; in the remaining 4 patients, one parameter was improved. Time-related efficacy parameters for all 37 patients were updated as of March 2004. Median follow-up was 41 months (range, 26-53 months); median time to progression was 6.6 months (range, 0.5-29.2 months; Fig. 1); and median OS was 10.7 months (range, 0.5-36.2 months; Fig. 2). One-year survival was $40 \%$ (Fig. 2).

\section{Toxicities}

A total of 149 cycles were administered (mean, 4 cycles per patient; range, $0-6$ ), with $6.0 \%$ of cycle delays being caused by adverse events (only $1.3 \%$ for hematologic toxicity and 1 cycle because of vomiting). Overall, the regimen was well tolerated. All patients (ITT population) were evaluated for toxicity (Table 3 ). WHO grade 3-4 febrile neutropenia occurred in 8 patients $(22 \%)$ and in $6 \%$ of cycles. Grade 3-4 neutropenia occurred in 1 patient $(3 \%)$ and in $1 \%$ of cycles. The most frequent grade 3-4 toxicities were alopecia, diarrhea, and vomiting, which occurred in $11 \%, 4 \%$, and $2 \%$ of cycles, respectively. Grade 1-2 asthenia and fatigue were observed in $43 \%$ of cycles. Grade 3 stomatitis occurred in 1 patient (3\%), grade 3 vomiting in 3 patients $(8 \%)$, and grade $3-4$ diarrhea in 5 patients $(14 \%)$. One patient had reversible grade 4 gastrointestinal bleeding. Two patients $(5 \%)$ died as a result of febrile neutropenia and sepsis. Four $(10.8 \%)$ patients withdrew from the study due to adverse events. The early (60-day) mortality rate was $10.8 \%$ (4 patients).

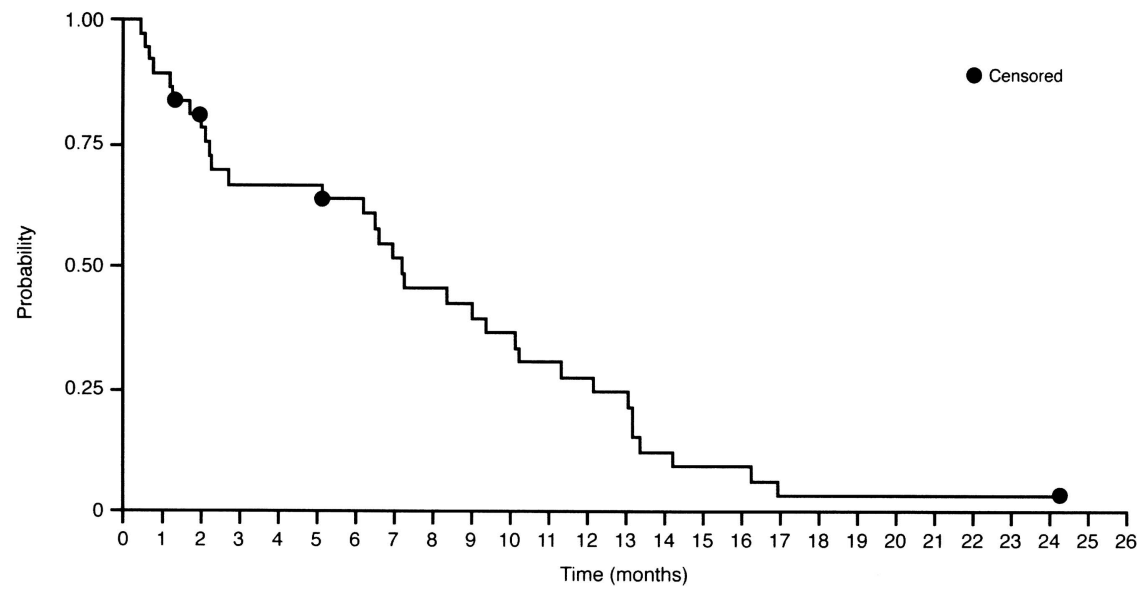

Fig. 1. Time to disease progression 


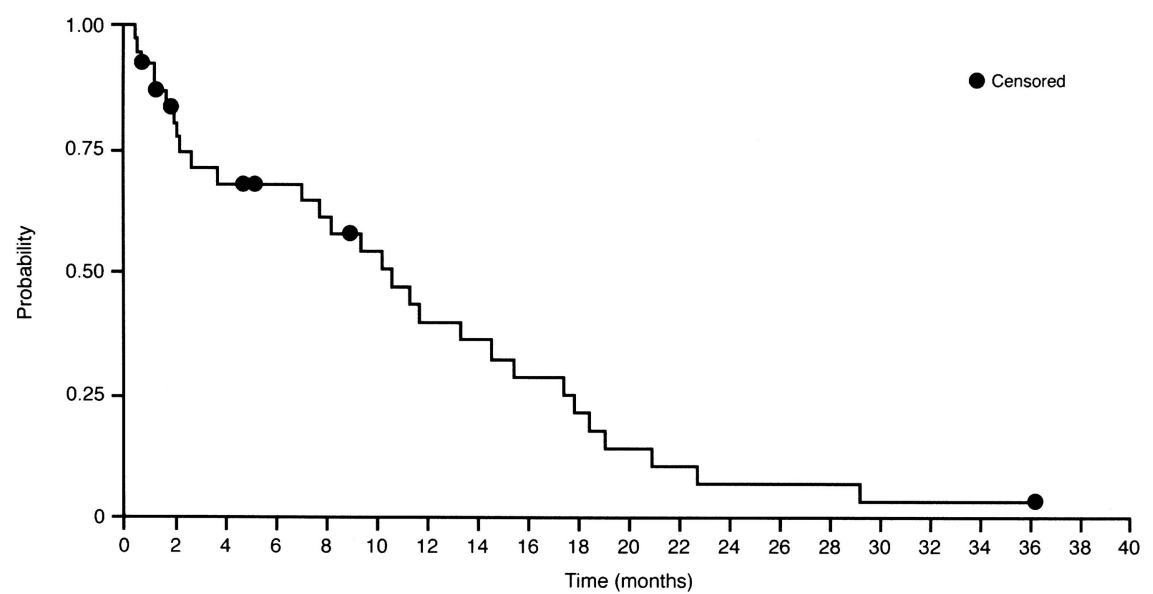

Fig. 2. Overall survival

Table 3. Toxicity, expressed as percentage of cycles $(n=149)$

\begin{tabular}{lrr} 
& \multicolumn{2}{c}{ NCI-CTC grade } \\
\cline { 2 - 3 } Toxicity & 3 & 4 \\
\hline Vomiting & 2 & 0 \\
Alopecia & 11 & 0 \\
Stomatitis & 1 & 0 \\
Diarrhea & 3 & 1 \\
Peripheral edema & 0 & 0 \\
Neuropathy & 0 & 0 \\
Myalgia & 0 & 0 \\
Infection & 1 & 0 \\
Neutropenia & 1 & 0 \\
Febrile neutropenia & 3 & 3 \\
Thrombocytopenia & 1 & 0 \\
Bleeding & 0 & 0 \\
Anemia & 1 & 0 \\
Asthenia/fatigue & 0 &
\end{tabular}

NCI-CTC, National Cancer Institute Common Toxicity Criteria ${ }^{a}$ NCI-CTC version 2.0

\section{Discussion}

Although gastric adenocarcinoma is the most chemosensitive gastrointestinal tumor, very few studies have shown improvement in patient survival or quality of life with chemotherapy [6]. The introduction of FAM at the end of the 1970s raised the possibility that combination chemotherapy could be more effective than a single agent. However, Cullinan et al. [36] demonstrated, in a comparative study, that FAM was not superior to 5-FU alone in terms of OS. Second-generation regimens, such as FAMTX, ELF, EAP, and ECF, have achieved an objective response in up to $51 \%$ of patients and a complete response in $10 \%-17 \%$ of patients. A median OS of 7-10 months and 2-year survivals of 5\%$10 \%$ have also been demonstrated $[3,6]$.
The taxanes, docetaxel and paclitaxel, may provide a treatment option that potentially improves efficacy and survival among patients with gastric cancer. Paclitaxel shows moderate activity as single-agent therapy [14-16] and shows good activity when combined with 5-FU: a response rate of $65.5 \%$, complete response rate of $24 \%$, and median OS of 12 months (range, 2-30+ months) have been reported in one study with this combination [23]. Furthermore, clinical benefit based upon weight gain, PS improvement, and analgesic consumption reduction was noted in $51.7 \%$ of patients [23]. However, these results need to be interpreted with care, because they were not obtained from an ITT analysis. Other studies have reported overall response rates of $32 \%$ with paclitaxel-5-FU-folinic acid [30] and 48\%-51\% with paclitaxel-5-FU-cisplatin with or without folinic acid $[29,37]$.

Docetaxel is also active in advanced gastric cancer as single-agent therapy [17-22]. For example, in a phase II European Organisation for Research and Treatment of Cancer (EORTC) trial [17], 37 eligible patients with advanced, untreated gastric carcinoma were given docetaxel $100 \mathrm{mg} / \mathrm{m}^{2}$ as a 60 -min IV infusion once every 3 weeks. Of the 33 evaluable patients, 8 (24\%) achieved partial remission for a median of 7.5 months, and 11 patients had stabilization of disease. Docetaxel has also been tested in combination with other agents. Preliminary results of a phase II study suggest response rates of $37 \%$ with docetaxel-cisplatin-5-FU (DCF) compared with $25 \%$ with epirubicin-cisplatin-5-FU (ECF) and $18.5 \%$ with docetaxel-cisplatin (DC) [38]. Moiseyenko and colleagues [39] conducted a phase III multinational study comparing DCF with the doublet cisplatin-5-FU (CF; V325), early results of which suggested the superiority of DCF over CF in patients with advanced gastric cancer. Updated interim results of this study also suggest that DCF may provide significantly higher response 
rates than $\mathrm{CF}[40,41]$. In total, 463 patients were randomized to two treatment arms: DCF given every 21 days (docetaxel $75 \mathrm{mg} / \mathrm{m}^{2}$ and cisplatin $75 \mathrm{mg} / \mathrm{m}^{2}$ on day 1 and 5-FU $750 \mathrm{mg} / \mathrm{m}^{2}$ per day [continuous IV infusion] for 5 days) and CF given every 28 days (cisplatin $100 \mathrm{mg}$ / $\mathrm{m}^{2}$ on day 1 and 5 -FU $1000 \mathrm{mg} / \mathrm{m}^{2}$ per day [continuous IV infusion] for 5 days). DCF was superior to CF in terms of objective response rate $(38.7 \%$ vs $23.2 \%$, respectively; $P=0.012$ ), median time to disease progression (5.2 months vs 3.7 months, respectively; $P=$ $0.0008)$, and median OS (10.2 months vs 8.5 months, respectively; $P=0.0064)[40,41]$. Notably, the probability of survival at 1 year with DCF was $44.1 \%$ [40].

The study of moiseyenko and colleagues [39] is the largest prospective, randomized phase III study to date in patients with advanced gastric cancer. It lends strong support for the use of docetaxel in the systemic therapy of patients with gastric cancer. However, there was a greater incidence of grade 3-4 neutropenia, febrile neutropenia, and neutropenia with infection in the DCFtreated patients compared with $\mathrm{CF}$. While the incidence of diarrhea was higher among DCF-treated patients, the risk of nausea, vomiting, and stomatitis was greater in patients treated with $\mathrm{CF}$ [41]. These findings raise the question of whether cisplatin is the best agent to use in combination with docetaxel and 5-FU.

In our investigation, which, as far as we are aware, is the first published study of DEF in gastric cancer the toxicity profile of epirubicin was better than might be expected with cisplatin. Epirubicin has activity in advanced gastric cancer equivalent to that of 5-FU [42] and, in gastric cancer cell lines, epirubicin seems to be more effective than doxorubicin [31]. Response rates, median time to progression, median OS, and 1-year survival with DEF were equivalent to those for DCF (35\% vs $38 \%, 6.6$ months vs 5.2 months, 10.7 months vs 10.2 months, and $40 \%$ vs $44.1 \%$, respectively). However, the toxicity profile of DEF appears to be better: grade 3-4 neutropenia, vomiting, stomatitis, and diarrhea occurred more frequently in DCF- than in DEFtreated patients $(84 \%$ vs $5 \%, 15 \%$ vs $8 \%, 23 \%$ vs $3 \%$, and $20 \%$ vs $14 \%$, respectively), although a direct comparison between studies cannot be made. It must be stated, however, that blood count was checked only at 21-day intervals. This may explain the low incidence of neutropenia observed with our regimen. Another advantage of the DEF regimen is the use of bolus 5-FU instead of continuous infusion, removing the requirement for infusion pumps. It is also important to point out that - based on analgesic consumption, weight gain, and ECOG PS improvement - DEF produced a clinical benefit in 11 patients $(30 \%)$.

The results obtained in this study indicate that DEF could be an alternative to DCF for the treatment of advanced gastric cancer, based on possible equivalent efficacy and a better toxicity profile, in addition to the easier mode of 5-FU administration. A phase III trial has been planned to further confirm the advantages of the DEF regimen.

Acknowledgments The authors thank Aventis Pharma for providing all medications utilized in this trial and for the statistical support (Ana Truzzi and Thais Cocarelli).

\section{References}

1. Ho D. Epidemiologic studies in gastric cancer. In: Ho D, editor. Gastric cancer. New York, NY: Churchill Livingstone; 1988. p. 125.

2. Ministry of Health, Brazil. National Secretariat of Health Assistance. National Câncer Institute of Brazil. Cancer Control Program Coordination. - Pro-Onco. Estimates of Cancer: Incidence and Mortality in Brazil, 2002. Rio de Janeiro, Brasil. Available from: http://www.inca.gov.br/ (accessed May 14, 2004).

3. Findlay M, Cunningham D. Chemotherapy of carcinoma of the stomach. Cancer Treat Rev 1993;19:29-44.

4. Rubin J, Gallagher JG, Schroeder G, Schutt AJ, Dalton RJ, Kugler JW, et al. Phase II trials of 5-fluorouracil and leucovorin in patients with metastatic gastric or pancreatic carcinoma. Cancer 1996;78:1888-91.

5. Louvet C, De Gramont A, Demuynck B, Nordlinger B, Maisani JE, Lagadec B, et al. High-dose folinic acid, 5-fluorouracil bolus and continuous infusion in poor-prognosis patients with advanced measurable gastric cancer. Ann Oncol 1991;2:229-30.

6. Murad AM. Chemotherapy for advanced gastric cancer: focus on new agents and combinations. Cancer Control 1999;6:361-8.

7. Kim NK, Park YS, Heo DS, Suh C, Kim SY, Park KC, et al. A phase III randomized study of 5-fluorouracil and cisplatin versus 5-fluorouracil, doxorubicin, and mitomycin $\mathrm{C}$ versus 5fluorouracil alone in the treatment of advanced gastric cancer. Cancer 1993;71:3813-18.

8. Wils J, Klein HO, Wagener DJ, Bleiberg H, Reis H, Korsten F, et al. Sequential high-dose methotrexate and fluorouracil combined with doxorubicin: a step ahead in the treatment of advanced gastric cancer - a trial of the European Organization for Research and Treatment of Cancer Gastrointestinal Tract Cooperative Group. J Clin Oncol 1991;9:827-31.

9. Cocconi G, Bella M, Zironi S, Algeri R, Di Costanzo F, De Lisi V, et al. Fluorouracil, doxorubicin, and mitomycin combination versus PELF chemotherapy in advanced gastric cancer: a prospective randomized trial of the Italian Oncology Group for Clinical Research. J Clin Oncol 1994;12:2687-93.

10. Kelsen D, Atiq OT, Saltz L, Niedzwiecki D, Ginn D, Chapman D, et al. FAMTX versus etoposide, doxorubicin, and cisplatin: a random assignment trial in gastric cancer. J Clin Oncol 1992; 10:541-8.

11. Vanhoefer U, Rougier P, Wilke H, Ducreux MP, Lacave AJ, Van Cutsem E, et al. Final results of a randomized phase III trial of sequential high-dose methotrexate, fluorouracil, and doxorubicin versus etoposide, leucovorin, and fluorouracil versus infusional fluorouracil and cisplatin in advanced gastric cancer: a trial of the European Organization for Research and Treatment of Cancer Gastrointestinal Tract Cancer Cooperative Group. J Clin Oncol 2000;18:2648-57.

12. Webb A, Cunningham D, Scarffe JH, Harper P, Norman A, Joffe $\mathrm{JK}$, et al. Randomized trial comparing epirubicin, cisplatin, and fluorouracil versus fluorouracil, doxorubicin, and methotrexate in advanced esophagogastric cancer. J Clin Oncol 1997;15:261-7.

13. Crown J, O'Leary M. The taxanes: an update. Lancet 2000;355: $1176-8$. 
14. Ajani JA, Ilson DH, Daugherty K, Pazdur R, Lynch PM, Kelsen DP, et al. Activity of Taxol in patients with squamous cell carcinoma and adenocarcinoma of the esophagus. J Natl Cancer Inst 1994;86:1086-91.

15. Ajani JA, Fairweather J, Dumas P, Patt YZ, Pazdur R, Mansfield PF. A phase II study of Taxol in patients with advanced gastric carcinoma. Cancer J Sci Am 1998;4:269-74.

16. Ohtsu A, Boku N, Tamura F, Muro K, Shimada Y, Saigenji K, et al. An early phase II study of a 3-hour infusion of paclitaxel for advanced gastric cancer. Am J Clin Oncol 1998;21:416-9.

17. Sulkes A, Smyth J, Sessa C, Dirix LY, Vermoken JB, Kaye S, et al. Docetaxel (Taxotere) in advanced gastric cancer: results of a phase II clinical trial. EORTC Early Clinical Trials Group. Br J Cancer 1994;70:380-3.

18. Einzig AI, Neuberg D, Remick SC, Karp DD, O’Dwyer PJ, Stewart JA, et al. Phase II trial of docetaxel (Taxotere ${ }^{\circledR}$ ) in patients with adenocarcinoma of the upper gastrointestinal tract previously untreated with cytotoxic chemotherapy: the Eastern Cooperative Oncology Group (ECOG). Med Oncol 1996;13:8793.

19. Mavroudis D, Kourousis C, Androulakis N, Kalbakis K, Agelaki $\mathrm{S}$, Kakolyris S, et al. Frontline treatment of advanced gastric cancer with docetaxel and granulocyte colony-stimulating factor (G-CSF): a phase II trial. Am J Clin Oncol 2000;23:341-4.

20. Taguchi T, Sakata Y, Kanamaru R, Kurihara M, Suminaga M, Ota J, et al. Late phase II clinical study of RP56976 (docetaxel) in patients with advanced/recurrent gastric cancer: a Japanese Cooperative Study Group trial (group A) (in Japanese). Jpn J Cancer Chemother 1998;25:1915-24.

21. Mai M, Sakata Y, Kanamaru R, Kurihara M, Suminaga M, Ota J, et al. A late phase II clinical study of RP56976 (docetaxel) in patients with advanced or recurrent gastric cancer: a Cooperative Study Group trial (group B) (in Japanese). Jpn J Cancer Chemother 1999;26:487-96.

22. Bang YJ, Kang WK, Kang YK, Kim HC, Jackes C, Zuber E, et al. Docetaxel $75 \mathrm{mg} / \mathrm{m}^{2}$ is active and well tolerated in patients with metastatic or recurrent gastric cancer: a phase II trial. Jpn J Clin Oncol 2002;32:248-54

23. Murad AM, Petroianu A, Guimaraes RC, Aragao BC, Cabral LO, Scalabrini-Neto AO. Phase II trial of the combination of paclitaxel and 5-fluorouracil in the treatment of advanced gastric cancer: a novel, safe and effective regimen. Am J Clin Oncol 1999; 22:580-6.

24. Kettner E, Ridwelski K, Keilholtz U, Gallkowski T, Gebauer H, Kroning K, et al. Docetaxel and cisplatin combination therapy for advanced gastric cancer: results of two phase II studies (abstract). Proc Am Soc Oncol 2001;20:165.

25. Ridwelski K, Gebauer T, Fahlke J, Kroning H, Kettner E, Meyer $\mathrm{F}$, et al. Combination chemotherapy with docetaxel and cisplatin for locally advanced and metastatic gastric cancer. Ann Oncol 2001;12:47-51.

26. Roth AD, Maibach R, Martinelli G, Fazio N, Aapro MS, Pagani $\mathrm{O}$, et al. Docetaxel (Taxotere) - cisplatin (TC): an effective drug combination in gastric carcinoma. Ann Oncol 2000;11:301-66.

27. Ajani JA, Fodor M, Van Cutsem E, Tjulandin S, Moiseyenco V, Cabral A, et al. Multinational randomized phase II trial of docetaxel and cisplatin with or without 5-fluorouracil in patients with advanced gastric cancer or GE junction adenocarcinoma (abstract). Proc Am Soc Clin Oncol 2000;19:247.

28. Cascinu S, Graziano F, Barni S, Labianca R, Comella G, Casaretti $\mathrm{R}$, et al. A phase II study of sequential chemotherapy with docetaxel after the weekly PELF regimen in advanced gastric cancer. A report from the Italian Group for the Study of Digestive Tract Cancer. Br J Cancer 2001;84:470-4.
29. Kim YH, Shin SW, Kim BS, Kim JH, Kim JG, Mok YJ, et al. Paclitaxel, 5-fluorouracil, and cisplatin combination chemotherapy for the treatment of advanced gastric cancer. Cancer 1999;85:295-301.

30. Bokemeyer C, Hartmann JT, Lampe CS, Clemens MR, Quietzsch D, Forkmann L, et al. Paclitaxel and weekly 24-hour infusion of 5fluorouracil/folinic acid in advanced gastric cancer. Semin Oncol 1997;24:S19-96-100.

31. Kohnoe S, Yoshida M, Takahashi I, Emi Y, Maehara Y, Sugimachi K, et al. Epirubicin is equivalent to adriamycin in vitro against many cancer cells but more effective against gastric cancer cells. Anticancer Res 1992;12:389-92.

32. CTC version 2.0. Cancer Therapy Evolution Program. Common Toxicity Criteria, version 2.0. DCTD, NCI, NIH and DHS, March 1998.

33. World Health Organization (WHO). Handbook for reporting results of cancer treatment. Geneva: WHO Offset publication No. 48; 1979.

34. Andersen JS, Burris HA, Casper E, Green MR, Rothemberg ML, Modiano MR, et al. Development of a new system for assessing clinical benefit for patients with advanced pancreatic cancer (abstract). Proc Am Soc Clin Oncol 1994;13:461.

35. Laurén P. The two histological main types of gastric carcinoma: a diffuse and so-called intestinal type carcinoma. Acta Pathol Microbiol Scand 1965;64:31-49.

36. Cullinan S, Moertel C, Fleming T, Rubin JR, Krook JE, Everson LK, et al. A comparison of three chemotherapeutic regimens in the treatment of advanced pancreatic and gastric carcinoma. JAMA 1985;253:2061-7.

37. Honecker F, Kollmannsberger C, Quietzsch D, Haag C, Schroeder M, Spott C, et al. Phase II study of weekly paclitaxel plus 24-h continuous infusion 5-fluorouracil, folinic acid and 3weekly cisplatin for the treatment of patients with advanced gastric cancer. Anticancer Drugs 2002;13:497-503.

38. Roth AD, Maibach R, Falk S, Stupp R, Saletti P, Kaberle D, et al. Docetaxel-cisplatin-5FU (TCF) versus docetaxel-cisplatin (TC) versus epirubicin-cisplatin-5FU (ECF) as systemic treatment for advanced gastric carcinoma (AGC): a randomized phase II trial of the Swiss Group for Clinical Cancer Research (SAKK). Proc Am Soc Clin Oncol 2004;23: abstract 4020. Updated data presented at the 2004 ASCO annual meeting (see www.ASCO.org for virtual meetings).

39. Moiseyenko V, Van Cutsem E, Tjulandin S, Tchao Y, Sengupta $\mathrm{S}$, Zuber E, et al. Docetaxel-cisplatin-5-FU (DCF) versus cisplatin-5-FU (CF) as first line therapy for gastric cancer: interim analysis results on efficacy and safety in a multicenter randomized phase III study (abstract). Proc Am Soc Clin Oncol 2002;21:147a.

40. Ajani JA, Van Cutsem E, Moiseyenko V, Tjulandin S, Fodor M, Majlis A, et al. Docetaxel (D), cisplatin, 5-fluorouracil compared to cisplatin $(\mathrm{C})$ and 5-fluorouracil $(\mathrm{F})$ for chemotherapy-naïve patients with metastatic or locally recurrent, unresectable gastric carcinoma (MGC): interim results of a randomized phase III trial (V325). Proc Am Soc Clin Oncol 2003;22:249 abstract 999; and associated slide presentation (www.asco.org/ac/1,1003,_12002511-00_18-0023-00_19-002584,00.asp).

41. Van Cutsem E. The treatment of advanced gastric cancer: new findings on the activity of the taxanes. Oncologist 2004;9 (Suppl 2):9-15.

42. Coombes RC, Chilvers CE, Amadori D, Medi F, Fountzilas G, Rauschecker $\mathrm{H}$, et al. Randomised trial of epirubicin versus fluorouracil in advanced gastric cancer. An International Collaborative Cancer Group (ICCG) study. Ann Oncol 1994;5:336. 